\title{
Synthesis of cholic acid-terminated dendritic lysine-block-poly[ethylene glycol]-block- dendritic lysine and its enhanced ability to solubilize Paclitaxel in water
}

\author{
Laurenzo D.V. Alba ${ }^{1^{*}}$, Ma. Luisa Enriquez ${ }^{2}$ \\ ${ }^{1}$ Chemistry Department, De La Salle University, 2401 Taft, Avenue, Manila 0922 \\ ${ }^{2}$ Biology Department, De La Salle University, 2401 Taft, Avenue, Manila 0922
}

\begin{abstract}
Paclitaxel, a chemotherapeutic drug used to inhibit mitosis in cancer cells, requires the use of the solubilizer Cremophor EL due to its poor water solubility. However, Cremophor EL is associated with adverse reactions following chemotherapy. This work reports the synthesis of an alternative solubilizing agent for paclitaxel. Two generations of dendritic lysine were coupled onto both ends of PEG-4000 via reaction of fluorenylmethyloxycarbonyl (FMOC)-lysine-FMOC-OH with dicyclohexylcarbodiimide (DCC)-mediated condensation, and deprotection with $30 \%$ piperidine. Cholic acid was attached to the terminal amino groups through the use of DCC/n-hydroxysuccinimide. Synthesis steps were monitored and confirmed by electrospray ionization mass spectroscopy. The results suggest that the synthesized polymer is a viable solubilizing agent for delivery of paclitaxel. Additional studies are required to assess its safety and stability.
\end{abstract}

Keywords: dendrimer; Lysine; PEG; paclitaxel; drug delivery

\section{INTRODUCTION}

Drug delivery is an active field of study that aims to improve the efficacy of pharmaceutical treatment by enabling the active compounds to reach their targets. However, it is a technical challenge if the drug is insoluble in the aqueous environment inside the body. Drug solubilization is a key step in the delivery of drugs that are generally non-polar. This can be achieved with the aid of (1) emulsifiers (chemicals that enable the dispersion of the drug into small droplets); (2) hydrotropic agents (chemicals that increase the water solubility of the drug), and/ or (3) micelles (spherical aggregates of amphipathic molecules) (Chadha et al., 2008). An amphipathic molecule consists of a hydrophilic, water-soluble portion, and a hydrophobic, lipid-soluble end. In micelles, molecules arrange themselves such that the hydrophilic portions extend outward to the aqueous environment of the body, concealing the hydrophobic portions of the molecule inside. It is possible for drugs to be loaded within the interior, increasing the bioavailability and allows for delivery to the target cells (Reul et al., 2011).

Paclitaxel $\left(\mathrm{C}_{47} \mathrm{H}_{51} \mathrm{NO}_{14}\right)$, an alkaloid derived from the yew (Taxus baccata), is a potent anticancer drug yet, its bioavailability in the body is problematic due to its low solubility in water. A common approach in the preparation of paclitaxel medication is through the addition of emulsifiers such as the Cremophor EL or polyethoxylated castor oil in the formulation. 
These emulsifiers however have been implicated in a number of adverse effects of chemotherapy. The chemotoxic effect of the emulsifiers calls for new approaches in the delivery of paclitaxel. Alternative strategies have been designed for paclitaxel delivery such as the use of microemulsions (Nornoo et al., 2008), copolymeric micelles (Li et al., 2013), hydrotropic agents (Cho et al., 2004), and dendrimers (Xiao et al., 2009). New approaches are still highly desired.

Polyethylene glycol (PEG), a polyether available in different molecular weights, presents a promising platform for drug solubilization. It is a generally hydrophilic molecule that, when covalently attached to drugs, increases their bioavailability and reduces their removal from the system. It is nontoxic at high molecular weights, and also has the ability to mask compounds from the immune system. Researches using PEG are usually focused on modifying one or both ends of the long molecule to develop micelleor gel-forming compounds that can be used for drug delivery, tissue scaffolds, or enzyme immobilization (Almany and Seliktar, 2004). This work explores the dendritic approach in modifying PEG and capping with a lipophilic moiety for enhanced paclitaxel interaction.

\section{EXPERIMENTAL}

Materials. Poly (ethylene glycol) (PEG4000), fluorenylmethyloxycarbonyl (FMOC)protected L-lysine (FMOC-Lys-FMOC-OH), $N, N^{\prime}$-dicyclohexylcarbodiimide (DCC), n-hydroxysuccinimide (NHS), ethyl 2-cyano2-(hydroxyimino)acetate (Oxyma Pure ${ }^{\mathrm{TM}}$ ), piperidine, benzoylacetone (BZA), and 4-dimethylaminopyridine (DMAP) we purchased from Merck. Cholic acid was purchased from Alfa Aesar. Dichloromethane (DCM), anhydrous diethyl ether, and dimethylformamide (DMF) was purchased from JT Baker.

Scanning electron microscopy was measured on JEOL JSM-V Scanning Electron Microscope. A drop of polymer solution was frozen over a glass slide and air-dried. A thin layer of gold was sputter-coated onto the sample, which was then mounted using carbon tape. The infrared spectrum was analyzed using a Nicolet FT-IR Spectrophotometer. A small amount of the dried polymer sample was ground with potassium bromide $(\mathrm{KBr})$ powder, pelletized, and placed inside the FT-IR sample holder. Mass spectra were taken using a Bruker MicroTOF QII LC-MS/MS unit operating in direct injection mode with electrospray ionization (ESI).

\section{Synthesis of Cholic acid-terminated Dendritic Lysine-block-poly(etbylene glycol)-block-dendritic} Lysine. Synthesis of Lysine-terminated PEG. PEG4000 ( 1,1 equivalent) and DMAP (0.2 eq.) were dissolved together in DCM with stirring under ambient conditions. FMOC-Lys-FMOC-OH (2, 2.5 eq.) and Oxyma (2.5 eq.) were dissolved in 50:50 DCM/DMF. This solution was preactivated by adding a solution of DCC (2.5 eq.) dissolved in DCM and stirring for 5 minutes. The resulting mixture was added to the PEG-4000 solution and allowed to react for 6 hours under continuous stirring. The precipitate consisting of dicyclohexylurea (DCU) was removed via vacuum filtration. The modified PEG-4000 was then precipitated by adding cold anhydrous ether $(40 \mathrm{~mL})$ and refrigerating for 10 15 minutes. The solvents and unreacted reagents were removed via centrifugation. The polymer was recrystallized by dissolving in a small amount of DMF and re-precipitating in cold ether, followed by centrifugation and drying the pellet at room temperature in a desiccator to afford 3.

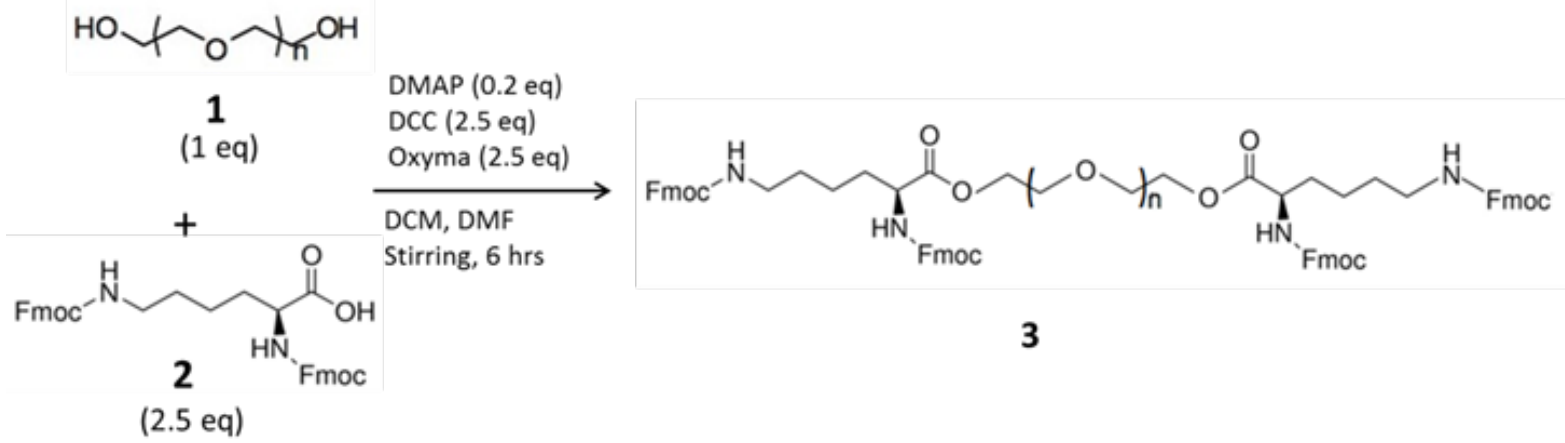

The Fmoc protecting group of $\mathbf{3}$ was removed by dissolving the polymer in $30 \%(\mathrm{v} / \mathrm{v})$ piperidine in DMF $(10 \mathrm{~mL})$ and letting stand for 30 minutes. Subsequently, diethyl ether was added to precipitate the polymer, followed by centrifugation and decanting. The polymer was recrystallized in diethyl ether twice, followed by drying to afford Gen 1 polymer. 
Following the steps described above, another round of coupling was carried out on the polymer to synthesize the second generation of dendritic lysine (Gen 2). The polymer synthesized in the first step took the place of PEG-4000; DMAP was not used anymore, and the amounts of all other reagents were doubled. Coupling and deprotection steps were monitored via ninhydrin test and ESI-mass spectrometry.

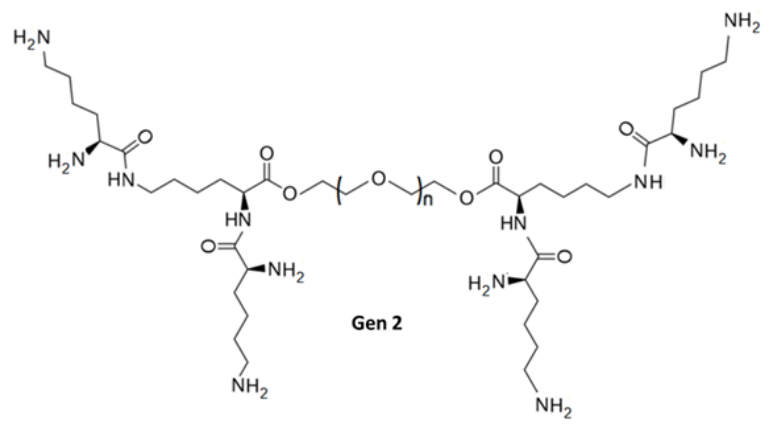

Capping the 2nd Generation PEG-dendritic Lysine with Cholic Acid. The caping moiety cholic acid n-hydroxysuccinimide (NHS) ester was first synthesized by dissolving cholic acid (10 eq.) in 50:50 DCM/DMF together with n-hydroxysuccinimide (10 eq.). DCC (10 eq.) in DCM was added while mixing, and allowed to react for 12 hours (Lapidot et al., 1967). The precipitate was filtered off, and the resulting solution was added to the Gen 2 polymer dissolved in a small amount of DCM with continuous stirring for 48 hours at ambient conditions. The polymer was then precipitated by adding diethyl ether and further recrystallized twice with diethyl ether.

The polymer synthesis was monitored by Electrospray ionization mass spectrometry (ESI-MS) using very small samples of PEG-4000, Gen 1, Gen 2 , and cholic acid-capped dendrimers dissolved in methanol (about $0.1 \mathrm{mg}$ in $10 \mathrm{~mL}$ ). Triethylamine was added to a final concentration of $0.1-0.3 \%(\mathrm{v} / \mathrm{v})$ as a charge stripping agent (Gudihal et al. 2012a). Mass spectra were collected and summated over 2 minutes in positive mode. Data were processed via Bruker Compass Data Analysis software. Manual deconvolution was performed by identifying polymer peaks and multiplying the masses by Z, ( $\mathrm{n}$ is rounded off to a whole number) according to the equation:

$$
Z=\frac{44.05}{\text { distance }(\mathrm{amu}) \text { between two polymer peaks }} \quad \text { Eq. } 1
$$

where 44.05 corresponds to the mass of one $\mathrm{CH}_{2} \mathrm{CH}_{2} \mathrm{O}$ - unit in poly (ethylene glycol), and $Z$ is the computed charge (Gudihal et al. 2012).

\section{Determination of Critical Micelle Concentration}

$(C M C)$. Aqueous solutions containing threefold serial dilutions starting with $0.99 \mathrm{mg} / \mathrm{mL}$ of the polymer were mixed with concentrated benzoylacetone dissolved in DMSO for a final concentration of $7.0 \times 10^{-5} \mathrm{M}$ benzoylacetone. The mixtures were vortexed and left to stand for 10 minutes at room temperature to allow the benzoylacetone to equilibrate. The absorbances of the solutions corresponding to peaks at or near 312 and $250 \mathrm{~nm}$ were recorded against blank samples containing the same amount of polymer but without benzoylacetone. The absorbance ratio A312/A250 was plotted versus log polymer concentration. The point at which the slope of the curve changes abruptly was taken as the critical micelle concentration, data which supports the formation of polymeric micelles (Dominguez et al. 1997).

\section{RESULTS AND DISCUSSION}

There are many different ways of modifying PEG for micelle formation and drug delivery. This study explored the possibility of attaching polylysine dendrimers to both ends of high molecular weight PEG. Previous studies have confirmed the feasibility of synthesizing branched polylysine from fluorenylmethyloxycarbonyl (FMOC) modified lysine in order to create regularly branched polylysine (Xiao et al., 2009). These were attached to both hydroxyl ends of the PEG molecule, whose particular reaction has been outlined by a previous study on the anti-inflammatory drug Ibuprofen (Nayak and Jain 2011).

Poly (ethylene glycol) 4000 (PEG-4000) is commercially available as a mixture of different oligomers with a molar mass range that attains an approximately normal distribution (Gudihal 2012). Mass spectral analysis of PEG 4000 reveals that the average mass is not exactly $4000 \mathrm{Da}$. The exact mass range was determined to be between 3276 - 5216 $\mathrm{Da}$ centered at $4158 \mathrm{Da}$. This average mass was used for all succeeding calculations of expected mass.

For the synthesis of lysine-based dendritic polymers, and for peptide synthesis in general, it is necessary to utilize $N$-protected amino acids to ensure that only one amino acid is added to exposed amines or hydroxyls at each coupling step. Fluorenylmethyloxycarbonyl (Fmoc) is one of the popular amine-protecting groups used in peptide synthesis. It is removed via the addition of a base, typically piperidine. The Fmoc group is liberated as dibenzofulvene, which further reacts with piperidine to form a piperidine-dibenzofulvene adduct. Without piperidine, the dibenzofulvene may irreversibly react with any exposed amine (such as 
those of lysine), permanently preventing any further coupling reaction. (Houben-Weyl 2002, AAPPTEC 2009).

In the synthesis procedure employed in this work, the addition of Oxyma was used to increase yield and to reduce the formation of $\mathrm{N}$-acylisourea, which complicates the purification process. The acyl group from the DCC-activated $\mathrm{O}$-acylisourea is transferred to Oxyma before finally being transferred to the target amine or hydroxyl, causing a slower yet more reliable reaction (Houben-Weyl 2002, AAPPTEC 2009).

During the first coupling, two equivalents of Fmoclys-fmoc-OH were esterified onto both ends of PEG-4000, with the loss of two water molecules. This increased the mass by $1145.3 \mathrm{Da}$, which is consistent with the observed mass spectrum (Figure $1)$. The mass spectrum of 3 shows the exact mass range to be 4464 - $6356 \mathrm{Da}$, centered at $5370 \mathrm{Da}$. The spectrum shows that the compound has gained $1212 \mathrm{Da}$ from the first coupling due to the addition of two Fmoc-lys-fmoc-OH (MW = 590.67 each) and removal of $2 \mathrm{H}_{2} \mathrm{O}(\mathrm{MW}=18.02)$ molecules. A gain of $1145.3 \mathrm{Da}$ is expected. The discrepancy between the theoretical and observed measurements is due to the contribution of $\mathrm{Na}^{+}(\mathrm{MW}=22.99)$ and/or $\mathrm{K}^{+}(\mathrm{MW}=39.10)$ to the positive charge.

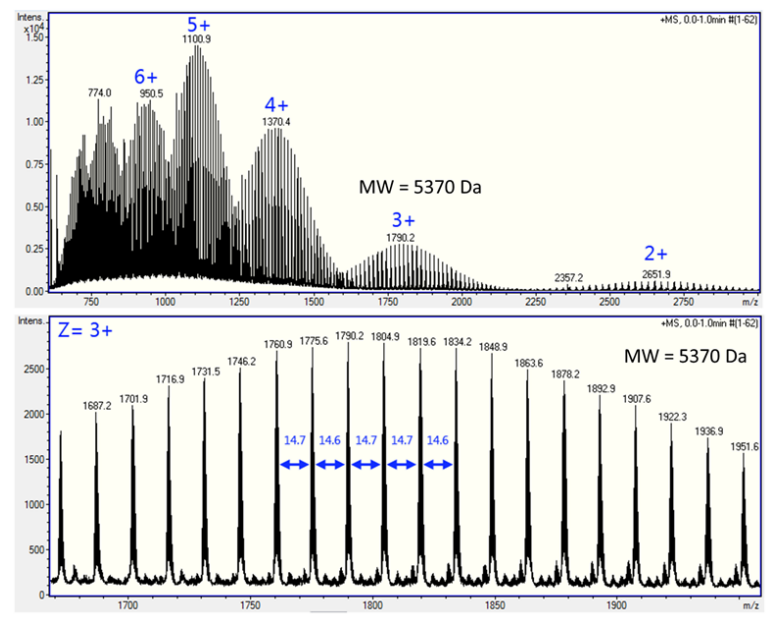

Figure 1. Mass Spectrum of 3, (Lysine-modified PEG4000 before Deprotection). Overview of the Mass Spectrum at $\mathrm{m} / \mathrm{z}=600-3000$ Showing the Multiple Charge Envelopes (top). Magnified View of the +3 Charged Polymer Signal (bottom).

Treating the compound $\mathbf{3}$ with piperidine removed four Fmoc groups (two from each lysine), causing a loss of 889 Da and exposed four reactive amine groups ready for the next coupling. Although it is theoretically possible to confirm the deprotection by measuring the loss of $889 \mathrm{Da}$, exposed amines interfere with the measurement with the electrospray ionization technique used in this study as observed in various trials with the instrument. Their tendency to be protonated makes the individual signal peaks cluster together, making it difficult to find the mass difference in related peaks and to apply equation (1). Instead, the success of the deprotection was measured with the mass spectrum taken after the next coupling step since an incompletelydeprotected compound will not react optimally with the next round of coupling and will produce a mass change significantly less (about $590 \mathrm{Da}$ ) than the predicted amount.

The second coupling step adds four Fmoc-lys-fmoc$\mathrm{OH}$ (2) to the exposed amine groups of Gen 1 with the loss of four water molecules. Unlike the previous step, this does not require addition of DMAP as amines are sufficiently reactive by themselves (Houben-Weyl 2002, AAPPTEC 2009). On the other hand, Oxyma participates in this reaction to stabilize the intermediate and prevent racemization. The reaction adds 2290.6 Da and creates the second generation of dendritic lysine. The expected mass from the step was also found to be close to the observed mass (Figure 2).

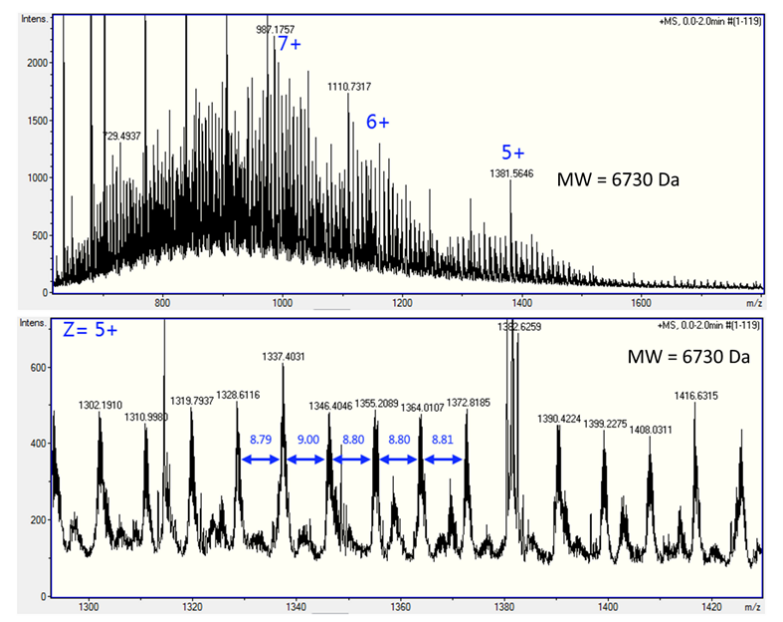

Figure 2. Mass Spectrum of Second Generation Lysine dendrimer-modified PEG-4000 before Deprotection. Overview of the Mass Spectrum at $\mathrm{m} / \mathrm{z}=600$ 1800 Showing the Multiple Charge Envelopes (top). Magnified View of the +5 charged Polymer Signal (bottom).

The mass spectrum of the polymer after the second coupling before deprotection with piperidine, shows the mass range to be about 6205-7525 Da, centered at about $6730 \mathrm{Da}$. The lower mass end was obliterated due to the overlapping +6 charge envelope. The spectrum shows that the compound gained $2287 \mathrm{Da}$ from the second coupling, which is still close to the predicted gain of 2290.6 Da. After this, the second deprotection step removed 8 Fmoc groups and replaced them with hydrogen, 
causing the loss of $1778 \mathrm{Da}$ to afford Gen 2. We attempted to synthesize the third generation of dendritic lysine before capping the dendrimer, however, the synthesized molecule exceeded the mass spectrometer limit of 10,000 Da. Thus, the synthesis of Gen 3 dendritic lysine cannot be verified by our instrument and thus was not further pursued.

The next reaction involves the addition of 8 cholic acid molecules onto the terminal amino groups in Gen 2 dendritic lysine. In theory, the use of DCC alone may be sufficient; however, literature recommends the use of n-hydroxysuccinimide (NHS) to form an activated ester with cholic acid (Xiao et al. 2009) in order to increase yield. The mechanism is like that of Oxyma-NHS, which causes the formation of a less labile intermediate that is still amine-reactive. The mass spectrum (Figure 3) of the cholic acid-terminated dendritic lysine-blockpoly(ethylene glycol)4000-block-dendritic lysine, after reaction with cholic acid NHS ester shows mass range of 5700-8883 Da. Additionally, two +3 charge envelopes were observed, with the first one ranging from 5700 to $7422 \mathrm{Da}$ (centered at about 6675 $\mathrm{Da}$ ) and 7494 to $8883 \mathrm{Da}$ (centered at about 8181 $\mathrm{Da})$. This confirms that the polymer gained up to $3934 \mathrm{Da}$ due to the addition of cholic acid. The +3 polymer charge envelope at $\mathrm{m} / \mathrm{z}=2200$ represents polymer molecules not completely reacted with cholic acid.

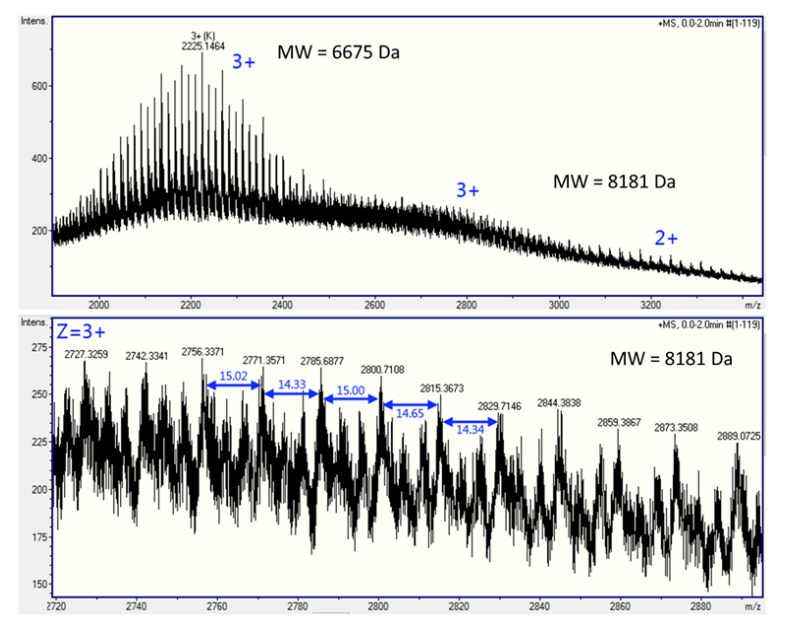

Figure 3. Mass Spectrum of the Final Cholic acidcapped Gen 2 polymer. Overview of the Mass Spectrum at $\mathrm{m} / \mathrm{z}=1900-3400$ Showing the Multiple Charge Envelopes (top). Magnified View of the +3 Charged Polymer Signal at $\mathrm{m} / \mathrm{z}=2800($ bottom $)$.

From the deconvoluted mass spectrum, it was found that the reaction with cholic acid-NHS ester was not complete, even after 48 hours reaction time. Each cholic acid has a mass of $408.57 \mathrm{Da}$. It is apparent that a minimum of 4 cholic acid molecules were successfully attached, with a smaller proportion being completely capped by cholic acid. The incomplete attachment of cholic acid suggests that steric effects became significant at this synthesis stage. The large size of the cholic acid substituent effectively "hides" the unreacted amine groups from the reactive NHS-cholic acid molecules.

In theory, the use of a significant excess cholic acid and DCC, such as 2 equivalents per hydroxyl or amine, will negate some of the steric hindrance (Niederhafner et al. 2006). However, as determined in our experimentation, cholic acid also has limited solubility in diethyl ether. This complicates the removal of excess cholic acid in the reaction mixture. Thus, only 1.25 equivalents of cholic acid per amine was used as a compromise between purity and reaction completion. Unfortunately, the completion of the reaction could not be enhanced further in this study. Purification steps were limited to repeated ether wash and recrystallization, which effectively removes most reactants not bound to the PEG- 4000 backbone. Cholic acid and its NHS ester most likely will be completely removed by several washings of diethyl ether. A total of $105 \mathrm{mg}$ of product was recovered and purified via recrystallization at the end of the synthesis phase. Initially, $3030 \mathrm{mg}$ of PEG-4000 was used. At four steps in the synthesis phase, only half of the product in the previous step was used. The maximum theoretical yield is 367 $\mathrm{mg}$. Thus, the cumulative percent yield of 5 reaction steps and 10 recrystallization steps was $28.6 \%$.

The successful overall synthesis of the dendritic polymer was also supported by the infrared spectrum. FTIR analysis (Figure 4) revealed the presence of all the possible functional groups of the expected synthesized polymer structure. The structurally relevant peaks are listed in Table 1 . The IR spectrum also indicated that the last deprotection step prior to cholic acid attachment was not $100 \%$ complete.

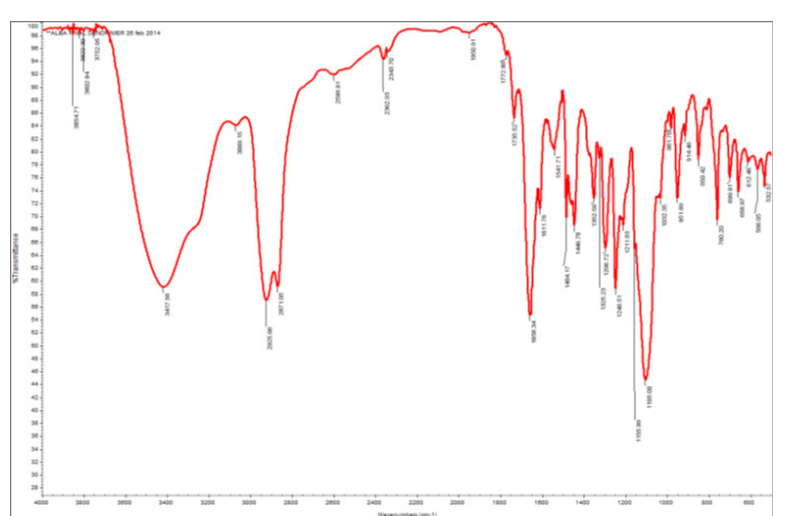

Figure 4. Infrared Spectrum of the Synthesized Polymer.

A similar copolymer was synthesized by Agrawal et al. (2007) using PEG-1000 and $t$-butyloxycarbonyl $(t$-BOC) lysine for the delivery of chloroquine phosphate for malaria treatment with promising 
Table 1. Interpretation of Observed Peaks in the Infrared Spectrum.

\begin{tabular}{llll}
\hline $\begin{array}{l}\text { Observed } \\
\text { peak }\left(\mathbf{c m}^{-1}\right)\end{array}$ & $\begin{array}{l}\text { Reference } \\
\text { wavenumber } \\
\left.\text { range } \mathbf{( c m}^{-1}\right)\end{array}$ & Identity & $\begin{array}{l}\text { Functional } \\
\text { group in } \\
\text { compound }\end{array}$ \\
\hline $\begin{array}{l}3417.56 \\
\text { (strong, broad) }\end{array}$ & $\begin{array}{l}3500-3200 \\
3500-3300\end{array}$ & $\begin{array}{l}\text { O-H stretch } \\
\mathrm{N}-\mathrm{H} \text { stretch }\end{array}$ & $\begin{array}{l}\text {-OH (cholic } \\
\text { acid) } \\
-\mathrm{NH}_{2} \\
\text { (unmodified } \\
\text { lysine) }\end{array}$ \\
\hline $\begin{array}{l}3069.15 \\
\text { (weak) }\end{array}$ & $3100-3010$ & =C-H stretch & $\begin{array}{l}\text { Attached } \\
\text { fluorine } \\
\text { (Fmoc) }\end{array}$ \\
\hline $\begin{array}{l}2926.66 \\
\text { (strong) }\end{array}$ & $2950-2850$ & Alkyl C-H & $\begin{array}{l}\text { Alkyl } \\
\text { backbone }\end{array}$ \\
2871.05 & stretch & $\begin{array}{l}\text { Ester (PEG- } \\
\text { Lys) }\end{array}$ \\
\hline $\begin{array}{l}1735.52 \\
\text { (sharp, weak) }\end{array}$ & $1750-1730$ & $\begin{array}{l}\text { Ester C=O } \\
\text { stretch }\end{array}$ & $\begin{array}{l}\text { Amide } \\
\text { (Lys-Lys, Lys- } \\
\text { Cholic acid) }\end{array}$ \\
\hline $\begin{array}{l}1656.34 \\
\text { (sharp, strong) }\end{array}$ & $1680-1640$ & Amide C=O \\
& & stretch & $\begin{array}{l}\text { PEG ether } \\
\text { backbone }\end{array}$ \\
\hline $\begin{array}{l}1105.00 \\
\text { (sharp, strong) }\end{array}$ & $1200-1000$ & C-O stretch \\
\hline
\end{tabular}

results. Another study by Xiao et al. (2009) investigated the possibility of encapsulating paclitaxel in a telodendrimer based on mPEG-5000$\mathrm{NH}_{3}$ and (Fmoc)Lysine(Fmoc)OH also showed promising ability for drug delivery. Micellar forms of the title dendrimer is expected to also show good promise in delivering drugs.

The formation of the micelles were confirmed by scanning electron micrograph of the surface of a thin film of the dried dendritic polymer (Figure 5). Evident on the image are numerous, spheroidal bumps that are characteristic of dried micelles. It shows prominent roundish bumps that are a characteristic of micelle formation (Du et al. 2010). Each structure ranged in diameter from 200-800 nm.

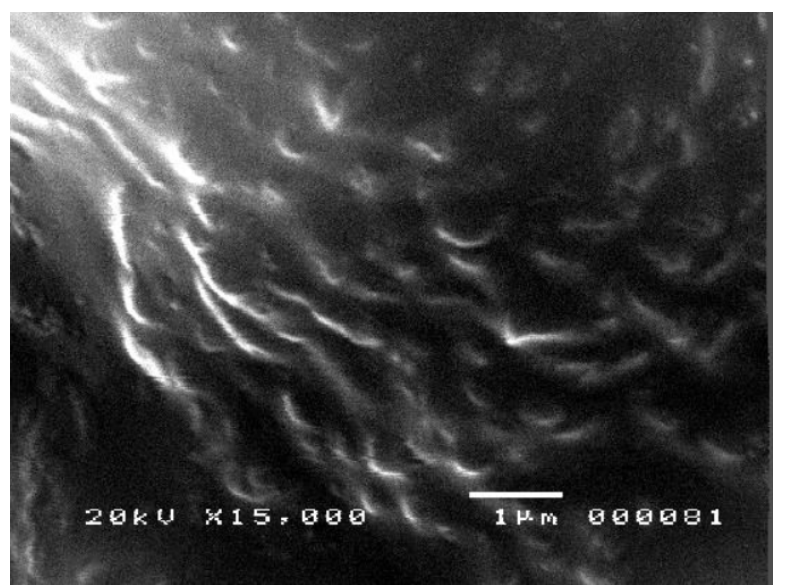

Figure 5. Scanning Electron Micrograph of the Polymer Sample Dried onto a Glass Plate.
The formation of micelles in a solution can be confirmed by the keto-enol tautomerization of benzoylacetone (1-phenyl-1,3-butanedione). This chemical, which is normally solid at room temperature and sparingly soluble in water, switches between two forms in a highly solvent-dependent manner. In aqueous or highly polar and protic environments, benzoylacetone exists predominantly as the keto form. Under these conditions, the enol cannot form due to intermolecular hydrogen bonding (i.e. with water). In nonaqueous environments, the enol form predominates due to intramolecular hydrogen bonding (Dominguez et al. 1997). The keto and enol forms have different UV absorption maxima at 250 and $312 \mathrm{~nm}$, respectively. As the concentration of the surfactant increases beyond the critical micelle concentration, the absorption at 312 $\mathrm{nm}$ is massively increased, and the absorption at 250 $\mathrm{nm}$ is diminished. This can be monitored by UV-Vis spectroscopy; the curve of absorbance at $312 \mathrm{~nm}$ can be plotted versus $\log$ [surfactant], and the inflection point is designated as the CMC (Dominguez et al. 1997). Figure 6 plots the ratio of absorbance at 312 $\mathrm{nm}(\mathrm{A} 312)$ and at $250 \mathrm{~nm}$ (A250), versus the log of the concentration of the polymer. The point where the slope changes abruptly was interpolated using the slopes of two lines. At the intersection of the extrapolated lines, the concentration of the polymer was found to be $0.244 \mathrm{mg} / \mathrm{mL}$. This value was taken to be the polymer's critical micelle concentration.

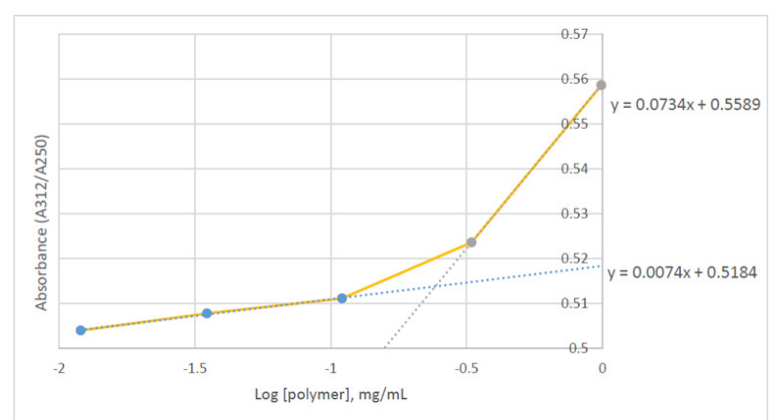

Figure 6. Benzoylacetone Assay Plot.

From the mass spectrum data, the molar CMC ranges from $2.74 \times 10^{-5}$ to $4.28 \times 10^{-5} \mathrm{M}$. However, the benzoylacetone assay is not optimal at this concentration range; the absorbance augmentation at $312 \mathrm{~nm}$ depends on the total amount of benzoylacetone incorporated into the forming micelles. If the critical micelle concentration is very low, the benzoylacetone uptake may be insignificant due to the very small number of micelles formed (Dominguez et al. 1997). The nonlinear increase in A312/A250 with respect to log of polymer concentration is highly indicative of the formation of micelles or micelle-like aggregates supporting the SEM data. A higher concentration of micelleforming surfactants will cause the formation of 
hydrophobic spaces where the benzoylacetone can migrate into, resulting in the observed spectrophotometric changes.

A characteristic of the resulting dendritic molecule is that it is a symmetrical block copolymer, where there are no polar and nonpolar ends. Instead, the central part of the molecule, the PEG-4000 moiety, is highly water-soluble while the terminal cholic acid groups are nonpolar. In polar solvent the dendritic polymer forms into micelles. Considering that the synthesized dendrimer can have a complete and/or partial capping, the dendiritic configurations (Fig $7 \mathrm{~A}$ and $\mathrm{B}$ ) are proposed. The number of cholic acid residues may be any number between 4 and 8 , and may assume any position. Possible aggregations in water are proposed to occur via micelles (Fig 7C) and/or polymersomes (Fig 7D) (Zhang et al. 2012).

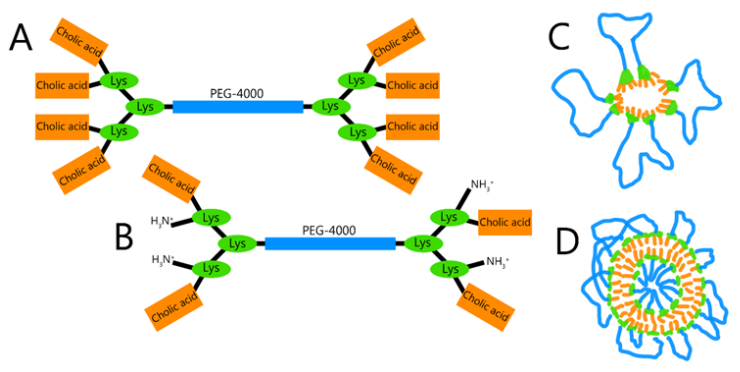

Figure 7. Schematic Diagram of the Synthesized Polymer $(A, B)$ and Its Possible Conformations in Water $(C, D)$.

After the synthesis of the title dendrimer, the resulting polymer was tested for its property to solubilize paclitaxel. The amphiphilic nature of the title dendrimer and its ability to form micelles allows it to interact both with polar and non-polar moeities. The anticancer drug paclitaxel has poor solubility in water at 8.5 to $17.1 \mu \mathrm{g} / \mathrm{mL}$ ("Paclitaxel MSDS", 2012) making it highly problematic in terms of bioavailability. The use of the title dendimer prepared in this work showed that the solubility of paclitaxel increased to $379.09 \mu \mathrm{g} / \mathrm{mL}$ - 22 fold increase in solublity! The solubilizing effect is attributed to the overall structure of the polymer. The terminal cholic acid groups function as lipophilic carriers that optimally interact with paclitaxel (Xiao et al. 2009). The PEG-4000 moiety is highly watersoluble, making the molecule itself an amphiphilic dendrimer that can accommodate paclitaxel to a high extent. The synthesized copolymer itself is water-soluble in all concentrations tested, up to 10 $\mathrm{mg} / \mathrm{mL}$.

The use of dendritic block copolymers carries inherent benefits in the design of targeted therapies. The title dendritic polymer is very promising due to its inherent large capacity for solubilization of the model drug. Moreover, its micellar amphiphilic character allows it to potentially accumulate in cancerous tissue due to the enhanced permeability and retention effect in the blood supply. The risks of toxicity can potentially be minimized since polylysine-based dendrimers are degraded by the body. This is in contrast to inorganic or metallic nanoparticles, which may persist indefinitely and cause a number of cytotoxic effects in normal tissues (Matsumura and Maeda, 1986).

\section{CONCLUSION}

The synthesis of poly(ethylene glycol) (PEG4000) grafted with second generation, cholic acidcapped dendritic polylysine through carbodiimidemediated coupling procedures was described. The final product was confirmed by mass spectrometry and infrared spectroscopy. A benzoylacetone assay and scanning electron microscopy of the polymer suggests that it is able to form micelles. Results showed that the title dendritic polymer was able to exhibit a 22-fold enhancement in the solubilization of pactitaxel in water making it a highly potential system in the delivery of paclitaxel and related drugs.

\section{REFERENCES}

[AAPPTEC] Advanced Automated Peptide Protein Technologies. Synthesis Notes Louisville, Kentucky. 2009 Available from: http://www.aapptec.com/ custdocs/aapptec $\% 20$ Synthesis $\% 20$ Guide $\% 20$ $2-0 \% 20 \% 282 \% 29$.pdf.

Almany L, Seliktar D. Biosynthetic hydrogel scaffolds made from fibrinogen and polyethylene glycol for 3D cell cultures. Biomaterials. 2004; 26(15):24672477. Available from: http://www.sciencedirect. com/science/article/pii/S014296120400609X

Agrawal P, Gupta U, Jain NK. Glycoconjugated peptide dendrimersbased nanoparticulate system for the delivery of chloroquine phosphate. Biomaterials, 2007; 28:3349-3359.

Chadha R, Kapoor VK, Thakur D, Kaur R, Arora P, Jain DVS. Drug carrier systems for anticancer agents: A review. J Scie Ind Res. 2008; 67(3):185197.

Cho YW, Lee J, Lee SC, Huh KM, Park K. Hydrotropic agents for study of in vitro paclitaxel release from polymeric micelles. J Control Release. 2004; 97(2):249-257. Available from: http://dx.doi. org/10.1016/j.jconrel.2004.03.013.

Dominguez A, Fernandez A, Gonzalez N, Iglesias 
E, Montenegro L. Determination of critical micelle concentration of some surfactants by three techniques. J Chem Educ. 1997; 74 (10):12271231. Available from: http://depa.fquim.unam.mx/ fina/nuevo/cmc.pdf

Du Z, Pan S, Yu Q, Li Y, Wen Y, Zhang W, Feng $\mathrm{M}, \mathrm{Wu}$ C. Paclitaxel-loaded micelles composed of folate-poly(ethylene glycol) and poly([gamma]benzyl l-glutamate) diblock copolymer. Colloid Surface A. 2010; 353:140-148. Available from: http://www.sciencedirect.com/science/article/pii/ S0927775709006554

Gudihal R, Babu S, Tang N, Palaniswamy S, Umamaheswari S, Basingi S. Analysis of PEG and a PEGylated Therapeutic Protein using an Agilent HPLC-Chip Coupled to a Q-TOF Mass Spectrometer. Agilent Application Note. 2012a. Available at http://www.lcms-connect.com/uploads/ tx_phdpagilentmediacenter/5991-1492EN.pdf

Gudihal R, Babu S, Tang N, Palaniswamy S, Umamaheswari S, Basingi S. Analysis of Polyethylene Glycol (PEG) and a Mono and Di-PEGylated Therapeutic Protein Using HPLC and Q-TOF Mass Spectrometry. Agilent Application Note. 2012b. Available at http://www.lcms-connect.com/uploads/ tx_phdpagilentmediacenter/5991-1509EN.pdf

Houben-Weyl. Thieme-Chemistry E22; c2002 [cited 2014 Feb 22]. Available from: http://www. thieme-chemistry.com/fileadmin/Thieme/HW100/pdf/april/Podlech_ZAW.pdf

Lapidot Y, Rappoport S, Wolman Y. Use of esters of $\mathrm{N}$-hydroxysuccinimide in the synthesis of $\mathrm{N}$-acylamino acids. J Lipid Res. 1967; 8:142-145. Available from: http://www.jlr.org/content/8/2/142. full.pdf

Li Y, Xu X, Shen Y, Qian C, Lu F, Guo S. Preparation and evaluation of copolymeric micelles with high paclitaxel contents and sustained drug release. Colloid Surface A. 2013; 429:12-18. Available from: http://dx.doi.org/10.1016/j.colsurfa.2013.03.043.

Nayak A, Jain A. In vitro and in vivo study of poly(ethylene glycol) conjugated ibuprofen to extend the duration of action. Sci Pharm. 2011; 79(2):359373. Available from: http://www.ncbi.nlm.nih.gov/ pmc/articles/PMC3134853/\#!po=3.33333

Niederhafner P, Šafařík M, Šebestík J, Gut V, Maloň P, Hlaváček J. Synthesis of protected peptides from the human IgG1 hinge region on PEG support using disulfide bond synthons and alkaline or enzymatic detachment. Tetrahedron Lett. 2006; 47(6):1023-
1025. Available from: http://dx.doi.org/10.1016/j. tetlet.2005.11.135

Nornoo A, Osborne D, Chow D. Cremophorfree intravenous microemulsions for paclitaxel: I: Formulation, cytotoxicity and hemolysis. Int. J. Pharma. 2008; 349(1-2):108-116. Available from: http://www.sciencedirect.com/science/article/pii/ S0378517307006461

Paclitaxel MSDS (Material Safety Data Sheet). LC Laboratories; c2012. Available from: http://www. lclabs.com/MSDS/P-9600MSDS.php4

Reul R, Renette T, Bege N, Kissel T. Nanoparticles for paclitaxel delivery: A comparative study of different types of dendritic polyesters and their degradation behavior. Int J Pharm. 2011; 407(1-2):190-196. Available from: http://www.sciencedirect.com/ science/article/pii/S0378517311000597

Xiao K, Luo J, Fowler W, Li Y, Lee J, Xing L, Cheng RH, Wang L, Lam K. A self-assembling nanoparticle for paclitaxel delivery in ovarian cancer. Biomaterials. 2009; 30(30):6006-6016. Available from: http://www.sciencedirect.com/science/article/ pii/S0142961209007200

Zhang L, He Y, Ma G, Song C, Sun H. Paclitaxelloaded polymeric micelles based on poly $(\varepsilon-$ caprolactone)-poly(ethylene glycol)-poly( $\varepsilon$ caprolactone) triblock copolymers: in vitro and in vivo evaluation. Nanomed-Nanotechnol. 2012; 8 (6):925-934. Available from: http://dx.doi. org/10.1016/j.nano.2011.11.005. 